\title{
A Severe Presentation of Drug-Induced Lupus Erythematosus
}

\author{
Ana Rita Nogueira ${ }^{\mathrm{a}, \mathrm{c}}$, Miguel Tabuas-Pereira ${ }^{\mathrm{a}}$, Antonio Aragao ${ }^{\mathrm{b}}$, \\ Manuel Teixeira Verissimo ${ }^{a}$
}

\begin{abstract}
Drug-induced lupus erythematosus is a rare condition associated with the exposure to certain drugs capable of triggering an autoimmune disease similar to systemic lupus erythematosus. Although there are no available diagnostic criteria, clinical and serological findings and its temporal association with the initiation of the suspected drug are important to establish the diagnosis. The withdrawal of the drug usually resolves the symptoms. However, in some cases, therapy with corticosteroids and immunosuppressive agents may be required. We present a 74-year-old female with a bilateral venous thrombosis, ischemic stroke, polyserositis and diarrhea due to drug-induced lupus erythematosus that required immunosuppressive therapy.
\end{abstract}

Keywords: Ischemic stroke; Drug-induced lupus; Autoimmunity; Systemic lupus erythematosus; Anti-histone antibodies

\section{Introduction}

The association between certain drugs and the triggering of autoimmune diseases or antibody production is well known from decades ago. Several drugs have been described to induce a condition similar to systemic lupus erythematosus in patients with no previous history of autoimmune diseases [1]. The first case was described in 1945 associated with treatment with sulfadiazine [2], although later it was considered a hypersensitivity reaction [3]. Since then, more than 90 drugs have been related with drug-induced lupus erythematosus (DILE) and the number is continuously increasing [1]. The majority of the drugs are classified as having a low or very low-risk profile to cause DILE, due to the paucity of cases described

Manuscript submitted August 8, 2019, accepted August 16, 2019

${ }^{a}$ Faculdade de Medicina, Universidade de Coimbra; Centro Hospitalar e Universitario de Coimbra, Coimbra, Portugal

${ }^{b}$ Centro Hospitalar e Universitario de Coimbra, Coimbra, Portugal

${ }^{\mathrm{c} C}$ Corresponding Author: Ana Rita Nogueira, Faculdade de Medicina, Univer-

sidade de Coimbra; Centro Hospitalar e Universitario de Coimbra, Coimbra,

Portugal. Email: ritagnogueira@gmail.com

doi: https://doi.org/10.14740/jmc3352 and probably to the lack of prospective studies [4]. More recently, the introduction of the biological modulators, used in the treatment of neoplastic and autoimmune diseases, led to an increase of the DILE cases. Although it might be difficult to establish a clear diagnosis in patients treated with these drugs, because they may have a pre-existing autoimmune condition acting as a confounder, the risk associated with the development of antibodies (and eventually an autoimmune disease) seems significantly increased [3].

\section{Case Report}

A 74-year-old female patient was admitted to our Internal Medicine Ward after multiple hospitalizations during the past year. She complained about anorexia, asthenia and significant weight loss, developing slowly throughout the last months. At the beginning of the symptoms, she was hospitalized due to bilateral thrombosis of the internal saphenous vein. Subsequently, the patient developed chronic non-bloody diarrhea, ascites and bilateral leg edema. Her family also reported that the patient had recent cognitive deterioration, decreased visual acuity and episodes of fever.

Previously, she reported a good health condition despite having a past medical history of dyslipidemia. Her medication included subcutaneous enoxaparin as a therapy for venous thrombosis and atorvastatin, prescribed 2 years before for primary cardiovascular disease prevention. The physical examination was compatible with severe sarcopenia, ascites, leg edema and bilateral pleural effusion. We also identified a triphasic Raynaud's phenomenon. The neurologic examination revealed left homonymous hemianopia and right homonymous quadrantanopia, with anosognosia. The laboratory findings revealed elevation of C-reactive protein, normocytic anemia, mild thrombocytopenia and normal leukocyte counts. Furthermore, the autoimmunity screening detected a high titer of anti-nuclear antibodies $(1: 1,280)$, with a homogenous pattern, low levels of anti-double stranded DNA (anti-dsDNA) antibody $(8.67 \mathrm{IU} / \mathrm{mL})$ and high titers of anti-histone antibodies $(>200 \mathrm{U} / \mathrm{mL})$. Complement levels (C3 and C4) were decreased and antiphospholipid syndrome antibodies were absent. The stool examination was unable to identify parasites or common pathogenic bacteria and the colonoscopy with biopsies was unremarkable. An abdominal paracentesis was performed showing a low serum ascitesalbumin gradient. Furthermore, the screening for celiac and 
neoplastic diseases was negative. Finally, a cerebral computed tomography (CT) revealed a right temporal/occipital ischemic lesion and a left temporal/parietal/occipital ischemic lesion. The transcranial Doppler ultrasonography, serial electrocardiography and echocardiography found no etiological cause for the stroke.

The diagnosis of drug-induced lupus secondary to statins was assumed. The treatment with atorvastatin was discontinued and prednisolone was initiated at $1 \mathrm{mg} / \mathrm{kg}$ and gradually tapered. A favorable clinical response was registered in the first week but unfortunately, the patient died 1 month after due to severe pseudomembranous colitis.

\section{Discussion}

DILE affects predominantly older patients (mean age 59 years) [3] and both genders equally [1]. The disease may occur weeks to years after the initiation of the drug and the onset is often gradual [4]. Although different drugs may induce different forms of DILE and diagnostic criteria are unavailable [5], the most common manifestations are arthralgia, malaise, fever, myalgia, anorexia and weight loss. Cutaneous and major organ involvement is less common than in systemic lupus erythematosus [4].

The laboratory findings include an increased erythrocyte sedimentation rate and an elevated C-reactive protein [6]. Leukopenia is present in $5-25 \%$ of the cases, anemia is detected in up to $35 \%$ of the patients and thrombocytopenia is rare in the majority of the cases [4]. Hypocomplementemia may be present although is not common [7]. The presence of antinuclear antibodies is frequent and has been suggested as a diagnostic criterion. All fluorescence patterns for antinuclear antibodies may be present but the most frequent is homogeneous. On the other hand, high levels of anti-dsDNA antibodies are rare [8] and should raise doubts about the diagnosis. In fact, high titers of anti-histone antibodies in the absence of high levels of antidsDNA antibodies have been proposed as a major criterion [4].

In severe cases, the treatment should include corticosteroids or other immunosuppressive drugs. However, in most cases with the withdrawal of the causative agent symptoms are reversible within weeks or months, although the serologic abnormalities may resolve more gradually [5].

In the reported case, infectious and neoplastic causes were extensively excluded and eventually contributed to the delay of the definitive diagnosis. Although consensual diagnostic criteria are not available in the literature, the patient presented typical symptoms and laboratory abnormalities of DILE. Although 24-h Holter was not readily available to exclude a cardioembolic etiology for the stroke, the patient was monitored during several days and performed several electrocardiograms and no arrhythmia was identified. In this context, we can hypothesize the risk factors associated with systemic lupus erythematosus (SLE) to be the cause for the stroke and the bilateral venous thrombosis.

The atorvastatin was considered to be the provocative drug, due to the temporal relation between its administration and the presence of the symptoms. Additionally, immunosup- pressive therapy was mandatory in this patient due to the central nervous system involvement and the severity of the symptoms.

\section{Conclusions}

The incidence of DILE is higher in older ages; and the establishment of the diagnosis requires a high level of suspicion, frequently leading to a delay in the diagnosis. Iatrogenesis should always be considered when the etiological cause is not easily perceived. A temporal association between the initiation of the suspected drug and the onset of the symptoms might be difficult in some cases, as it might have existed for an interval of years. However, the resolution of the symptoms after the withdrawal of the drug is essential and confirms the diagnosis.

\section{Acknowledgments}

None to declare.

\section{Financial Disclosure}

None to declare.

\section{Conflict of Interest}

None to declare.

\section{Informed Consent}

Informed consent for publication was obtained.

\section{Author Contributions}

Ana Rita Nogueira and Miguel Tabuas-Pereira participated in drafting the article; Antonio Aragao and Manuel Teixeira Verissimo revised the paper critically for important intelectual content.

\section{Abbreviations}

CT: computed tomography; DILE: drug-induced lupus erythematosus; SLE: systemic lupus erythematosus

\section{References}

1. Araujo-Fernandez S, Ahijon-Lana M, Isenberg DA. Drug-induced lupus: Including anti-tumour necrosis factor and interferon induced. Lupus. 2014;23(6):545-553.

2. Aresu L, Zanatta R, Luciani L, Trez D, Castagnaro M. 
Severe renal failure in a dog resembling human focal segmental glomerulosclerosis. J Comp Pathol. 2010;143(23):190-194.

3. Chang C, Gershwin ME. Drug-induced lupus erythematosus: incidence, management and prevention. Drug Saf. 2011;34(5):357-374.

4. Borchers AT, Keen CL, Gershwin ME. Drug-induced lupus. Ann N Y Acad Sci. 2007;1108:166-182.

5. Rubin RL. Drug-induced lupus. Expert Opin Drug Saf.
2015;14(3):361-378

6. Schlienger RG, Bircher AJ, Meier CR. Minocyclineinduced lupus. A systematic review. Dermatology. 2000;200(3):223-231.

7. Vedove CD, Del Giglio M, Schena D, Girolomoni G. Drug-induced lupus erythematosus. Arch Dermatol Res. 2009;301(1):99-105.

8. Katz U, Zandman-Goddard G. Drug-induced lupus: an update. Autoimmun Rev. 2010;10(1):46-50. 